\title{
Pautas Básicas para una ImpLantación EXITOSA del Costeo Basado en Actividades (ABC)
}

\author{
Recepción: Febrero de 2005 / Aceptación: Junio 2005
}

(1) José Carrión Nin

\section{RESUMEN}

Este artículo busca establecer una guía práctica de los principales pasos a seguir, mostrando experiencias y dando sugerencias que ayudaran a quien pretenda implementar un efectivo Costeo Basado en Actividades. La implementación del $A B C$ requiere de un complejo y analítico proceso que es costoso y requiere de tiempo, es por eso que los administradores naturalmente desean estar seguros de las ventajas antes de haberse embarcado en la implementación del $\mathrm{ABC}$.

Palabras Clave: Costos basados en actividades. Inductor. Gestión basada en actividades.

Basic Guidelines for a Successful Implantation of the Activity Based Costing (ABC)

ABSTRACT

This article seeks to establish a practice guide of the main steps to follow, showing experiences and giving suggestions to the one pretending to implement an Activity Based Costing. Implementation of ABC requires a complex and analytical process that is expensive and timeconsuming, that is why managers may want to be sure of the advantages before embarking upon the implementation of $\mathrm{ABC}$.

Key words: Activity based costs. Inductor. Activity based management.

(1) Magister en Administración, Magíster en Contabilidad, y Gestión Pública. Ingeniero de Costos - SUNARP - Sede Lima.

E-mail: jcarrion_lima@sunarp.gob.pe
I N T ROD U C C I Ó N

Son casi dos décadas desde que en 1987 Robert S. Kaplan y Robin Cooper[2] creadores del ABC establecen este nuevo enfoque de calcular y gestionar los costos, herramienta indispensable para poder competir en el ambiente empresarial actual donde las organizaciones requieren contar con información sobre los costos y eficiencia de los procesos del negocio que les permita tomar decisiones empresariales en forma acertada y oportuna.

La metodología de costeo convencional presenta dos fases principales:

1. La primera fase de acumulación de los costos de los recursos insumidos en una clasificación natural o autodescriptiva de los mismos (cuentas contables de gastos), los cuales a su vez pueden ser directos o indirectos.

2. La segunda fase es la adjudicación de los costos indirectos, esta última fase del costeo convencional es la cuestionada por los seguidores del $A B C$. Con los costos directos no existen controversias por que su imputación es tangible y verificable.

El cuestionamiento en el costeo convencional en esta segunda fase es debido a que este utiliza bases de volumen, como horas hombre, horas máquina, kilogramos de producto terminado, etc. no tomando en cuenta el grado de dificultad en elaborar los productos, esto descalificaría la precisión del costeo convencional, según los seguidores de esta metodología.

El hecho que el costeo convencional utilice bases de volumen para la distribución de los costos indirectos no significa que no pueda considerar nuevas bases como los inductores de actividad y uso.

Una crítica que ha recibido la técnica de costeo $A B C$ por algunos es que no cumple con los principios contables; sin embargo, esto no es del todo cierto ya que por ejemplo versiones actualizadas del Plan Contable General[5] revisado aplicado para empresas peruanas plantea diseñar las cuentas costos con denominación de actividades en lugar de centros de costos de esta forma es posible efectuar la dinámica contable en función a actividades, tal como se hace otros países[6].

Pero la adopción de éstas nuevas bases de distribución en función a las actividades demandará inversión de recursos y tiempo que algunas organizaciones no están dispuestas a brindar.

$E I A B C$ requiere de dos consideraciones básicas y previas para su aplicación; una de ellas, es que la empresa haya diversificado sus líneas de 
productos y servicios en forma considerable y la segunda es que una porción importante de sus costos sean indirectos.

Existen empresas que han obtenido beneficios importantes derivados de una adecuada implantación del ABC; sin embargo, también existen compañías que debido a una mala administración del proyecto no han logrado reducir sus costos, incrementar sus utilidades, mejorar su desempeño o realizar cambio alguno.

El Ingeniero Industrial tiene la formación profesional y la orientación a la optimización de procesos que le permiten tomar el liderazgo en este tipo de proyectos, ya que maneja las estrategias de la empresa y puede generar y administrar información sobre los costos, la rentabilidad y el desempeño del negocio. Es por esto, que la promoción y administración de un proyecto de costos $A B C$ es una gran oportunidad para mejorar el posicionamiento del Ingeniero Industrial en los niveles directivos de la empresa.

MARCO CONCEPTUAL

¿Qué es el ABC y cómo puede utilizarse? $A B C$ son las siglas en inglés de "Activity Based Costing" que en español significa Costeo Basado en Actividades, siendo esta una metodología para asignar los costos y gastos de una empresa.

La metodología $A B C$ se basa en el hecho de que una empresa para producir requiere realizar actividades las cuales consumen recursos, por lo que primero se costean las actividades y luego el costo de las mismas es asignado a los diferentes objetos de costo (productos, servicios, grupos de clientes y regiones, etc.) que demandan dichas actividades; con esto se logra una mayor precisión en la determinación de los costos y la rentabilidad.

Apaza, sobre el ABC remarca: "La diferencia entre los sistemas tradicionales de asignación básica de costos y los sistemas $A B C$ consiste en que estos últimos se centran en la acumulación de costos en actividades clave, en tanto que la asignación tradicional de costos se centra en la acumulación de costos en unidades de la organización como departamentos." [1].

El alcance completo de ABC incluye las actividades de todos los procesos principales de la organización; sin embargo, puede iniciarse con las actividades de fábrica para obtener información para efectos financieros (valoración de inventarios por ejemplo) y luego pasar a las actividades de los demás procesos para obtener información para la toma de decisionesgerenciales.

¿Cómo saber si su empresa requiere el
costeo ABC y está en posibilidades de
acceder a él?

A continuación se describen algunos aspectos importantes que deben considerarse para evaluar si se justifica en una empresa la implantación de un sistema $A B C$ :

a. Existencia de sistema integrado funcionando adecuadamente

Para que una empresa pueda implantar el costeo $A B C$, primero debe contar con sistemas automatizados para el procesamiento y registro integral de sus operaciones, en virtud de que una gran cantidad de la información que es requerida por el costeo ABC proviene de datos estadísticos de subsistemas operativos, tales como Compras, Almacenes, Producción, Ventas, etc., así como de subsistemas financieros como Contabilidad General, Activos Fijos, Cobranza, etc.

Si la empresa no cuenta con un sistema integrado para la cuantificación de sus actividades primeramente debe dedicar sus esfuerzos a resolver este asunto.

b. Sistema de Costeo funcionando adecuadamente En el caso de empresas manufactureras se debe contar previamente con un costeo adecuado de los materiales y mano de obra directos, debido a que la mecánica de costeo de los mismos permanece intacta al adoptar la técnica ABC. Es decir, si una empresa costea por orden o proceso, en forma estándar o histórica bajo un esquema absorbente lo seguirá haciendo de igual manera aún después de utilizar $A B C$.

Lo único que cambiará con el $\mathrm{ABC}$ para efectos de costeo de productos, es la asignación de los gastos indirectos de fabricación a los productos o servicios que será en función a bases de actividad.

En una empresa de servicios los costos directos normalmente son mínimos, la mayor parte son indirectos, por lo que el ABC se aplica por "default".

c. Magnitud significativa en los gastos indirectos Si una empresa se encuentra con altos niveles de costos indirectos, se deberá considerar seriamente la aplicación de la técnica ABC.

Uno de los cambios importantes en el ambiente de manufactura ha sido la incorporación de maquinaria y equipo con tecnología avanzada, lo 
cual, en consecuencia, ha generado una reducción en los costos de mano de obra directa.

d. Alta diversidad de productos, clientes, canales de distribución y puntos de venta

Cuando una empresa fabrica en una misma planta diversas líneas y tipos de productos y costea bajo la técnica tradicional de asignación de gastos indirectos normalmente se incurre en subsidios cruzados entre los diferentes productos, porque la distribución se realiza con bases de volumen, las cuales no reflejan de manera adecuada la absorción de todos los recursos utilizados en la planta.

En resumen, podemos establecer que si una empresa tiene un sistema administrativo integral, un considerable nivel de costos indirectos y además existe una alta diversidad de productos, canales de distribución, puntos de venta, clientes, y la imperante necesidad de precisión respecto al costo unitario de producción, se deberá considerar seriamente la implantación el costeo ABC en su empresa.

ORGANIZACIÓN DEL PROYECTO

Si bien la implantación del ABC representa una oportunidad importante para mejorar la ventaja competitiva en las empresas, este tipo de proyectos conllevan a un alto grado de dificultad y riesgo.

Para el desarrollo de este proceso y reducción del riesgo aludido es recomendable realizar las siguientes actividades preliminares:

1. Designar a un grupo de profesionales, integrado por personas que tengan el respaldo absoluto de la alta dirección y con amplia experiencia en los procesos de la empresa y en administración de costos. Este grupo será el responsable de la organización, planeación y administración del proyecto hasta su implantación.

2. Capacitar al grupo profesional en la metodología ABC para su implantación.

Una vez que se cuente con el grupo base adecuadamente capacitado para el desarrollo del proyecto $A B C$, será responsabilidad de éste, promover el proyecto en toda la organización.

Es común que muchas empresas candidatas para utilizar un sistema $A B C$ aún no lo hayan implantado debido a que la alta dirección y la gerencia no han entendido su importancia y otorgan mayor prioridad a otros proyectos como incrementar la capacidad instalada en ciertas líneas de producción, abrir nuevas plantas y nuevos centros de distribución, invertir en el desarrollo de nuevos productos, entre otros. Lo que desconocen es que al no contar con la información que proporciona $A B C$ pueden estar invirtiendo en productos, clientes o canales de distribución no rentables y al mismo tiempo afectando el desarrollo de otros que si lo son.

DEFINICIÓN DEL ALCANCE Y ENFOQUE
DEL PROYECTO
Alcance

\section{Procesos de negocio}

En primer lugar se debe establecer qué procesos del negocio se van a incluir y cuál es la secuencia de actividades que conviene seguir. Para esta tarea es recomendable utilizar el enfoque de "Análisis de Cadenas de Valor para crear y mantener ventaja competitiva" desarrollado por el profesor Michael Porter. Bajo este enfoque los procesos de la Cadena de Valor son los que intervienen directamente en el producto o servicio que se ofrece al cliente mientras que los procesos de apoyo brindan servicios en forma indirecta a la Cadena de Valor. Si bien este concepto es válido para las empresas en general, cada tipo de industria tiene una Cadena de Valor con aspectos particulares.

La secuencia típica para empresas manufactureras es iniciar definiendo los procesos de producción. Como siguiente paso podrían considerarse los procesos de logística, ventas, mercadotecnia y finalmente, dentro de los procesos de la Cadena de Valor del negocio, podrían incluirse los procesos de desarrollo de nuevos productos y servicio al cliente.

La última etapa continuaría definiendo los procesos de apoyo tales como administración de personal, administración financiera, servicios de asesoría legal, administración de sistemas y tecnología, administración de relaciones externas y administración corporativa, entre otros.

El alcance completo del $A B C$ comprende todos los procesos de la empresa, por lo que es común caer en la tentación de querer definir desde un inicio todos los procesos del negocio; sin embargo, con objeto de obtener resultados cuantificables del proyecto es preferible hacerlo en forma secuencial, es recomendable iniciar con uno de los procesos de la Cadena de Valor [3] del negocio e ir avanzando paulatinamente con cada uno de los otros procesos. 


\section{Productos y Zonas Geográficas.}

La definición del alcance del proyecto también deberá considerar qué productos y zonas geográficas se quieren incluir.

Es común que se pretenda incluir en un inicio todos los productos y zonas geográficas; sin embargo, de igual manera que en los procesos de negocio, es recomendable definir una estrategia de avance progresivo, ya que si se desea un enfoque global se requerirán demasiados recursos y el tiempo en el que se empiecen a recibir beneficios será mayor.

\section{Aplicaciones de la metodología ABC}

Las principales aplicaciones que se pueden tener con un sistema $A B C$ son:

- El costeo de actividades

- El costeo de productos

- La reducción de costos

- El mejoramiento de procesos

- La Administración del desempeño

- El presupuesto basado en actividades

Es normal que se quiera alcanzar la mayor cantidad de información a la vez; sin embargo, lo recomendable es establecer una secuencia de avance realista, de acuerdo con las prioridades de la empresa, e ir avanzando paulatinamente, hasta contar con toda la funcionalidad que se requiera.

Avanzar en forma gradual permitirá a la empresa asimilar y dar pasos firmes en la implantación del concepto ABC.

Enfoque

La definición del enfoque de un proyecto $A B C$ consiste en establecer la forma en la que se pretende llevar a cabo la implantación del sistema en términos del grado de integración, la periodicidad y amplitud de uso que hace con la información.

Las tres opciones de implantación del concepto ABC son:

\section{Proyecto Piloto}

Bajo este enfoque se realiza la implantación del sistema con un alcance limitado, el objetivo es ganar experiencia y comprobar que se pueden obtener beneficios para la empresa. El modelo es desarrollado en un paquete para PC monousuario con funciones básicas de costeo de actividades y productos. El sistema ABC no tiene interfaces automatizadas con los sistemas administrativos de la empresa. La información es estática y distribuida en forma limitada.

\section{Implantación permanente}

En esta opción de implantación se contemplan los principales procesos de la Cadena de Valor. Se utiliza un sistema más sofisticado para plataforma cliente servidor multiusuario que ya incluye otras aplicaciones tales como: Análisis de Rentabilidad, Presupuesto Basado en Actividades, Análisis de Capacidad Utilizada y Simulaciones, entre otras. El sistema ya cuenta con interfaces automatizadas con los sistemas administrativos y la información es actualizada mensualmente y se distribuye a algunos de los administradores operativos y financieros.

\section{Implantación totalmente integrada}

Bajo esta opción de implantación se contemplan todos los procesos de la empresa. El sistema está integrado en línea con los sistemas administrativos y la información es utilizada diariamente en las mayoría de las áreas de la empresa.

\section{¿Cuál de las tres opciones anteriores es la más} conveniente?

Lo conservador y cauto es pasar por cada una de estas fases en orden progresivo. Este enfoque también facilita el cambio en la cultura administrativa de la empresa, ya que la implantación de la metodología $A B C$ representa un cambio de la forma tradicional de administración por centro de costos a una administración basada en actividades que no es fácil de incorporar.

DISEÑO E IMPLANTACIÓN DEL SISTEMA

Una vez que la alta dirección y las gerencias operativas ya definieron el enfoque y alcance del proyecto $A B C$, se deben organizar los equipos de trabajo, capacitar al personal de la empresa, diseñar el modelo $A B C$, desarrollar y probar el sistema y por último implantarlo.

Capacitación $y$ entrenamiento en $A B C$ Para una adecuada capacitación y entrenamiento se deben realizar sesiones de capacitación y entrenamiento a los grupos de trabajo involucrados en el proyecto que cubran principalmente los siguientes aspectos:

1. Teoría sobre el concepto de ABC.

2. Información que se puede obtener y cómo se puede utilizar.

3. Cómo realizar el diseño del modelo.

4. Entrenamiento en el uso del software.

5. El proceso de implantación del sistema.

Los dos primeros temas deben ser talleres para todos los grupos involucrados, es decir, a los directo- 
res, gerentes y el equipo de trabajo; los puntos tres al cinco serán para el equipo de trabajo.

Análisis de importancia relativa de los costos

Es fundamental realizar un análisis de los costos de los procesos involucrados.

En este trabajo se debe incluir el análisis paretiano para identificar en que actividades se encuentra el $80 \%$ de los costos. Este análisis servirá para entender la estructura de costos de las operaciones de la empresa, y con esto tener bases para focalizar el diseño del modelo.

Diseño del modelo $A B C$

Las principales recomendaciones para el diseño de un modelo $A B C$ son:

\section{Definición de objetos de costo}

La definición de objetos de costo es fundamental para el diseño del modelo $A B C$, ya que establece las dimensiones de información que debe proporcionar el modelo. Esta definición dependerá del alcance que se quiera dar al sistema y las características de operación de la empresa, los objetos de costos pueden ser:

- Productos y línea de productos.

- Clientes o segmentos de clientes.

- Canal de distribución o ruta.

- Zona, región geográfica y país.

\section{Definición de actividades y Procesos}

Para la definición de actividades es recomendable considerar los siguientes aspectos:

- Utilizar el enfoque de la Cadena de Valor para determinar las actividades del negocio.

- Por otro lado: "Un proceso se define como una serie de actividades ligadas a fin de alcanzar un propósito específico". [4].

- Para efectos de lograr una adecuada precisión en el costeo de actividades es suficiente con llegar al tercer nivel en la definición de las mismas .

\section{Nivel Actividad}

1er Proceso de negocio: comercializar

2do Subproceso: vender

3er Tarea: levantar pedidos

4to Paso: capturar datos del cliente

5to Acción: capturar número de clientes

- Todas estas representan actividades en la empresa pero a un diferente nivel. Sólo para casos de reingeniería de procesos es necesario bajar del 3er nivel.
- Una actividad definida siempre debe tener un resultado tangible y cuantificable. Un modelo sólo debe definir aquellas actividades que las áreas encuentren relevantes y tratando de agrupar en ellas a todas las demás.

- Es frecuente que en los proyectos de $A B C$ se quiera definir las actividades de todos los procesos al máximo nivel de detalle, sin embargo, esto es un error porque sólo agrega complejidad al modelo y no genera ningún beneficio.

3. Definición de "drivers de costos"

- Un "driver" debe ser información disponible y cuantificable por objeto de costo.

- Una actividad debe tener sólo un "driver".

- Es importante distinguir entre "driver de recurso" (transportan el costo desde los recursos hasta las actividades) y "driver de actividad" (transportan el costo de las actividades hasta los productos o servicios).

Implantación

Las recomendaciones básicas para el proceso de implantación son:

- EI ABC, proporciona información valiosa para la toma de decisiones, pero los beneficios se obtienen siempre y cuando se lleven a cabo posteriores acciones correctivas.

- EI ABC debe estar conformando parte de los sistemas administrativos integrados.

- Se debe ver la organización como una serie de procesos integrados y administrar las actividades inherentes a cada proceso.

- El ABC debe estar integrado con los procesos de reingeniería en caso de estarse dando.

- El involucramiento de la alta dirección y la gerencia es necesario a lo largo de todo el proyecto para lograr una adecuada implantación.

C O N C L U S I Ó N

EI ABC aporta información empresarial útil para promover la ventaja competitiva en las empresas; sin embargo, no todas las empresas que emprenden un proyecto de implantación del $A B C$ logran concretar los beneficios cualitativos y cuantitativos que se pueden obtener.

Una implantación exitosa se debe en gran medida a la forma en que se desarrolla el proyecto y el compromiso de los funcionarios de más alto nivel en el éxito del mismo; además, del convencimiento de estos de que el $\mathrm{ABC}$ es una herramienta que representará una ventaja competitiva inminente. 
〉 > Pautas Básicas para una Implantación Exitosa del Costeo Basado en Actividades (ABC)

B I B L I O G R A F Í A

1. Apaza Meza, Mario. (2002). Costos $A B C, A B M y$ ABB Herramientas para incrementar la Rentabilidad y la Competitividad Empresarial. Pág. 65,348. Real Editores. Lima, Perú.

2. Bellido Sánchez, Pedro Alberto. (2000). Costos $A B C$. Pág. 63. Pacifico Editores. Lima, Perú.

3. Bravo Cervantes, Miguel H. (1999). Activity Based Costing. Pág. 211. Gráfica Filadelfia. 1era Edición

\section{Lima, Perú.}

4. Hansen y Mowen. (1995). Administración de Costos - Contabilidad y Control. Pág 272. Internacional Thomson Editores. S.A. México.

5. Paredes Reátegui, Villacorta Cavero. (2001). Plan Contable General Revisado. Págs. 922,923. Pacífico Editores E.I.R.L. Lima, Perú.

6. Sáez Torrecilla. (1997). Contabilidad de Costos. Mc Graw Hill. México. 